\title{
The Combinatorial Effects of Cognitive Behavioral Therapy with Aerobic Exercise in the Treatment of Fibromyalgia Bryan Ko', Matthew Bair ${ }^{2}$ \\ ${ }^{1}$ Indiana University School of Medicine; ${ }^{2}$ Regenstrief Institute
}

In the US alone, the chronic pain condition of fibromyalgia affects approximately 10 million people. Current treatment protocols focus heavily on pharmacological interventions; however, such treatments are unfavorable due to debilitating side effects and a lack of effectiveness in $2 / 3$ of patients. Two non-pharmacological interventions which have been shown to produce meaningful pain relief are Cognitive Behavioral Therapy (CBT) and Aerobic Exercise. However, the combinatorial effects of these treatments have never been fully explored and it is unknown if these treatments interact in an additive manner or if the benefits of one negate the benefits of the other. In this work we conducted a literature review using the databases of Pubmed, OVID MEDLINE, PsychInfo, and Embase from 1995 to May 2020 to determine if the combinatorial effects of these two treatments on pain relief and pain interference are significantly greater than that of aerobic exercise alone. We reviewed over 200 potential articles for relevance. Seven papers were found to directly address our target question, two from the US and five from Europe with sample sizes ranging from 34 to 442 . One paper showed improvement in pain intensity and two papers showed improvement in pain interference due to combinatorial treatment over aerobic exercise alone. When evaluating our results, we weighted these papers based on sample size, length of treatment and observation, method of CBT administration, and surveys chosen for data collection. We were unable to definitively show that CBT in combination with aerobic exercise is more effective than aerobic exercise alone at decreasing pain intensity and/or interference in patients with fibromyalgia. However, most of these papers identified several psychological and design based confounding factors that could influence their results. We recommend that more research be conducted in this area to further ascertain the effectiveness of this specific combinatorial treatment for fibromyalgia. 